\title{
GENERALIZED ANALYTIC INDEPENDENCE ${ }^{1}$
}

\author{
JACOB BARSHAY
}

\begin{abstract}
ABSTRACr. If a is a proper ideal of a commutative ring with unity $A$, a set of elements $a_{1}, \ldots, a_{n} \in A$ is called a-independent if every form in $A\left[X_{1}, \ldots, X_{n}\right]$ vanishing at $a_{1}, \ldots, a_{n}$ has all its coefficients in a. supa is defined as the maximum number of a-independent elements in $\mathbf{a}$. It is shown that grade $\mathbf{a} \leqslant \sup a \leqslant$ height $\mathbf{a}$. Examples are given to show that sup a need take neither of the limiting values and strong evidence is given for the conjecture that it can assume any intermediate value. Cohen-Macaulay rings are characterized by the equality of sup and grade for all ideals (or just all prime ideals). It is proven that equality of sup and height for all powers of prime ideals implies that the ring is $S_{1}$ (the Serre condition). Finally, independence is related to the structure of certain Rees algebras.
\end{abstract}

The notion of analytic independence relating sets of elements in a local ring to the maximal ideal of that ring can be delocalized. This generalization was made by Valla [4], [5] and leads to many interesting questions, several of which are considered here. Throughout this paper, "ring" will mean a commutative, Noetherian ring with unity.

Definition 1. If $a_{1}, \ldots, a_{n}$ are elements of a ring $A$ and $\mathbf{a}$ a proper ideal of $A$, then we say that $a_{1}, \ldots, a_{n}$ are a-independent if any form $F\left(X_{1}, \ldots, X_{n}\right)$ $\in A\left[X_{1}, \ldots, X_{n}\right]$ such that $F\left(a_{1}, \ldots, a_{n}\right)=0$ must have all of its coefficients in a.

Proposition 1. Let a be an ideal of $A, a_{1}, \ldots, a_{n}$ a set of a-independent elements. Then:

(1) If $n \geqslant 2$, then $a_{1}, \ldots, a_{n} \in \mathbf{a}$.

(2) If $\mathbf{b} \supseteq \mathbf{a}$, then $a_{1}, \ldots, a_{n}$ are $\mathbf{b}$-independent.

(3) If $\left\{a_{i_{1}}, \ldots, a_{i_{m}}\right\} \subseteq\left\{a_{1}, \ldots, a_{n}\right\}$, then $a_{i_{1}}, \ldots, a_{i_{m}}$ are a-independent.

(4) If $F\left(X_{1}, \ldots, X_{n}\right) \in A\left[X_{1}, \ldots, X_{n}\right]$ is a form of degree $s$ such that $F\left(a_{1}, \ldots, a_{n}\right) \in \mathbf{a}\left(a_{1}, \ldots, a_{n}\right)^{s}$, then $F\left(X_{1}, \ldots, X_{n}\right) \in \mathbf{a} A\left[X_{1}, \ldots, X_{n}\right]$.

Due to (1), we will assume henceforth that sets of a-independent elements come from a.

The notion of a-independence is related to the structure of the Rees algebra of the ideal generated by the set of elements. Recall that the Rees algebra of an ideal $\mathbf{a}$ in a ring $A$ is $\oplus_{r \geqslant 0} \mathbf{a}^{r}$ where $\mathbf{a}^{0}=A$. It is denoted by $R(\mathbf{a})$. If $\mathbf{a}=\left(a_{1}, \ldots, a_{n}\right)$, then

Received by the editors May 2, 1975.

AMS (MOS) subject classifications (1970). Primary 13C15, $13 \mathrm{H} 10$.

Key words and phrases. Analytic independence, grade, height, and sup of an ideal, CohenMacaulay ring, Rees algebra.

1 This work was supported by Grant No. 10164 of the C.U.N.Y. Faculty Research Award Program. 


$$
R(\mathbf{a}) \approx A\left[a_{1} t, \ldots, a_{n} t\right] \subset A[t] .
$$

Part (4) of the preceding proposition can be used to demonstrate the following result of Rees.

Proposition 2. Let $A$ be a ring, $\mathbf{a}=\left(a_{1}, \ldots, a_{n}\right)$ and $\mathbf{b} \supseteq \mathbf{a}$ ideals of $A$. Then $a_{1}, \ldots, a_{n}$ are $\mathbf{b}$-independent if and only if $R(\mathbf{a}) / \mathbf{b} R(\mathbf{a})$ is isomorphic to $(A / \mathbf{b})\left[X_{1}, \ldots, X_{n}\right]$.

Proof. Consider the exact sequence

$$
0 \rightarrow Q_{\infty}\left(a_{1}, \ldots, a_{n}\right) \rightarrow A\left[X_{1}, \ldots, X_{n}\right] \stackrel{\gamma}{\rightarrow} R(\mathbf{a}) \rightarrow 0
$$

where $Q_{\infty}\left(a_{1}, \ldots, a_{n}\right)$ is the homogeneous ideal generated by all forms which vanish at $a_{1}, \ldots, a_{n}$. Tensoring over $A$ with $A / \mathbf{b}$ gives

$$
Q_{\infty}\left(a_{1}, \ldots, a_{n}\right) \otimes_{A} A / \mathbf{b} \rightarrow(A / \mathbf{b})\left[X_{1}, \ldots, X_{n}\right] \rightarrow R(\mathbf{a}) / \mathbf{b} R(\mathbf{a}) \rightarrow 0 .
$$

But $a_{1}, \ldots, a_{n}$ are $\mathbf{b}$-independent if and only if

$$
Q_{\infty}\left(a_{1}, \ldots, a_{n}\right) \subseteq \mathbf{b} A\left[X_{1}, \ldots, X_{n}\right]
$$

so the result follows.

Definition 2. Let $A$ be a ring and a a proper ideal of $A$. Then sup a $=\sup \{n \mid \mathbf{a}$ contains $n$ a-independent elements $\}$.

In fact Valla has shown that sup a is bounded above and below by the height of $\mathbf{a}(\mathrm{ht} \mathbf{a})$ and the grade of $\mathbf{a}(\mathrm{gr} \mathbf{a})$, respectively. Thus sup a is just the maximum number of a-independent elements in a. For the sake of completeness, we include here a brief description of this work.

The fact that gra $\leqslant$ sup $\mathbf{a}$ is an immediate consequence of

Proposition 3. Let $A$ be $a$ ring and $a_{1}, \ldots, a_{n}$ an $A$-sequence. Then $a_{1}, \ldots, a_{n}$ are $\left(a_{1}, \ldots, a_{n}\right)$-independent.

Proof. It is well known ([1] or [3]) that for ideals generated by $A$-sequences, the symmetric algebra and the Rees algebra are isomorphic. In particular, $Q_{\infty}\left(a_{1}, \ldots, a_{n}\right)$ is generated by 1-forms $\sum_{i=1}^{n} b_{i} X_{i}$ such that $\sum_{i=1}^{n} b_{i} a_{i}=0$. But $a_{1}, \ldots, a_{n}$ being an $A$-sequence implies that $H_{1}\left(K\left(a_{1}, \ldots, a_{n} ; A\right)\right)=0$ where $K\left(a_{1}, \ldots, a_{n} ; A\right)$ denotes the Koszul complex. Thus every $b_{i} \in\left(a_{1}, \ldots\right.$, $\left.a_{n}\right)$, i.e., $Q_{\infty}\left(a_{1}, \ldots, a_{n}\right) \subseteq\left(a_{1}, \ldots, a_{n}\right) A\left[X_{1}, \ldots, X_{n}\right]$.

If $\mathbf{p}_{1}, \ldots, \mathbf{p}_{t}$ are the minimal primes of an ideal a of $A$, then sup a $\leqslant \sup \mathbf{p}_{i}$ for $i=1, \ldots, t$ by (2) of Proposition 1. Since ht a $=\min _{i=1, \ldots, t}\left\{\mathrm{ht} \mathbf{p}_{i}\right\}$, to show that $\sup \mathbf{a} \leqslant \mathrm{ht} \mathbf{a}$, it suffices to verify the inequality for prime ideals. For $A$ an integral domain, this result is due originally to Boger [2].

Proposition 4. Let $\mathbf{p}$ be a prime ideal in an integral domain $A$. Then $\sup \mathbf{p} \leqslant$ ht $\mathbf{p}$.

Proof. Let $\sup \mathbf{p}=n, a_{1}, \ldots, a_{n} \in \mathbf{p}$ a set of $\mathbf{p}$-independent elements, $\mathbf{a}=\left(a_{1}, \ldots, a_{n}\right)$. By Proposition 2 ,

$$
R(\mathbf{a}) / \mathbf{p} R(\mathbf{a}) \approx(A / \mathbf{p})\left[X_{1}, \ldots, X_{n}\right]
$$

so $\mathbf{p} R(\mathbf{a})$ is a prime ideal of $R(\mathbf{a})$ and $\mathrm{ht}(\mathbf{p} R(\mathbf{a})) \geqslant 1$ since $\mathbf{p} R(\mathbf{a}) \neq 0$. 
Furthermore $\mathbf{p} \subseteq \mathbf{p} R(\mathbf{a}) \subseteq \mathbf{p} A[t]$ and $\mathbf{p} A[t] \cap A=\mathbf{p}$ implies that $\mathbf{p} R(\mathbf{a}) \cap A$ $=\mathbf{p}$. Denote by $K$ the quotient field of $A$ and by $k$ the quotient field of $A / \mathbf{p}$. Applying Proposition 2, p. 326 of [6] gives

$$
\text { ht }(\mathbf{p} R(\mathbf{a}))+t d_{k} k\left(X_{1}, \ldots, X_{n}\right) \leqslant \mathrm{ht} \mathbf{p}+t d_{K} K(t)
$$

from which ht $\mathbf{p} \geqslant n$.

Proposition 5. Let $\mathbf{p}$ be a prime ideal of a ring $A$. Denote by $A_{\mathrm{red}}$ the reduction of $A$, i.e., $A / \sqrt{ } 0$ and by $\mathbf{p}_{\text {red }}$ the image of $\mathbf{p}$ in $A_{\text {red }}$. Then

(1) $\sup \mathbf{p}=\sup \left(\mathbf{p} A_{\mathbf{p}}\right)$,

(2) $\sup \mathbf{p}=\sup \mathbf{p}_{\text {red }}$.

Proof. (1) Observe that $R(\mathbf{a}) \otimes_{A} A_{\mathbf{p}} \approx R_{A_{\mathbf{p}}}\left(\mathbf{a} A_{\mathbf{p}}\right)$. Thus localizing the exact sequence

$$
0 \rightarrow I \rightarrow(A / \mathbf{p})\left[X_{1}, \ldots, X_{n}\right] \rightarrow R(\mathbf{a}) / \mathbf{p} R(\mathbf{a}) \rightarrow 0
$$

gives the exact sequence

$$
0 \rightarrow I_{\mathbf{p}} \rightarrow\left(A_{\mathbf{p}} / \mathbf{p} A_{\mathbf{p}}\right)\left[X_{1}, \ldots, X_{n}\right] \rightarrow R\left(\mathbf{a} A_{\mathbf{p}}\right) / \mathbf{p} R\left(\mathbf{a} A_{\mathbf{p}}\right) \rightarrow 0 .
$$

If $\sup \mathbf{p}=n, a_{1}, \ldots, a_{n}$ a set of $\mathbf{p}$-independent elements, $\mathbf{a}=\left(a_{1}, \ldots, a_{n}\right)$, then $I=0$. Thus $I_{\mathbf{p}}=0$ which gives $a_{1}, \ldots, a_{n}$ also $\mathbf{p} A_{\mathbf{p}}$ independent. Conversely, if $\sup \mathbf{p} A_{\mathbf{p}}=n$, it is clear that a set of $\mathbf{p} A_{\mathbf{p}}$-independent elements $a_{1}, \ldots, a_{n}$ can be chosen in $\mathbf{p}$. Then $I_{\mathbf{p}}=0$ which gives $I=0$. Thus $a_{1}, \ldots, a_{n}$ are $\mathbf{p}$-independent.

(2) Denote by $\bar{c}$ the image in $A_{\text {red }}$ of $c \in A$ and by $\bar{F}\left(X_{1}, \ldots, X_{n}\right)$ the image in $A_{\text {red }}\left[X_{1}, \ldots, X_{n}\right]$ of $F\left(X_{1}, \ldots, X_{n}\right) \in A\left[X_{1}, \ldots, X_{n}\right]$. If $a_{1}, \ldots, a_{n}$ are pindependent elements and $\bar{F}\left(\bar{a}_{1}, \ldots, \bar{a}_{n}\right)=\overline{0}$, then $F\left(a_{1}, \ldots, a_{n}\right)$ is nilpotent. Thus $F^{s}\left(X_{1}, \ldots, X_{n}\right) \in \mathbf{p} A\left[X_{1}, \ldots, X_{n}\right]$ for some $s \geqslant 1$ which gives

$$
F\left(X_{1}, \ldots, X_{n}\right) \in \mathbf{p} A\left[X_{1}, \ldots, X_{n}\right]
$$

and

$$
\bar{F}\left(X_{1}, \ldots, X_{n}\right) \in \mathbf{p}_{\text {red }} A_{\text {red }}\left[X_{1}, \ldots, X_{n}\right] .
$$

Thus $\bar{a}_{1}, \ldots, \bar{a}_{n}$ are $\mathbf{p}_{\text {red }}$-independent. Conversely, if $a_{1}, \ldots, a_{n} \in \mathbf{p}$ represent a set of $\mathbf{p}_{\text {red }}$-independent elements $\bar{a}_{1}, \ldots, \bar{a}_{n}$ and $F\left(a_{1}, \ldots, a_{n}\right)=0$, then $\bar{F}\left(\bar{a}_{1}, \ldots, \bar{a}_{n}\right)=\overline{0}$. Thus $\bar{F}\left(X_{1}, \ldots, X_{n}\right) \in \mathbf{p}_{\text {red }} A_{\text {red }}\left[X_{1}, \ldots, X_{n}\right]$ and $F\left(X_{1}, \ldots, X_{n}\right)$ has its coefficients in $\mathbf{p}+\sqrt{ } 0=\mathbf{p}$.

Since the equalities of Proposition 5 hold with sup replaced by ht, it suffices to consider the case of the maximal ideal in a reduced local ring.

Proposition 6. Let $A$ be a reduced local ring with maximal ideal $\mathbf{m}$. Then $\sup \mathbf{m} \leqslant$ ht $\mathbf{m}$.

Proof. Let $\mathbf{p}_{1}, \ldots, \mathbf{p}_{k}$ be the minimal primes of $A$ and consider the canonical map $A \rightarrow \oplus_{i=1}^{k} A / \mathbf{p}_{i}$ which is injective since $A$ is reduced.

By Proposition 4, sup $\mathbf{m} / \mathbf{p}_{i} \leqslant \mathrm{ht} \mathbf{m} / \mathbf{p}_{i} \leqslant \mathrm{ht} \mathbf{m}$ for each $i=1, \ldots, k$. Set ht $\mathbf{m}=n$ and let $a_{1}, \ldots, a_{n+1} \in \mathbf{m}$. For each $i=1, \ldots, k$, there exists a form $F_{i}\left(X_{1}, \ldots, X_{n+1}\right) \in A\left[X_{1}, \ldots, X_{n+1}\right]$ of degree $s_{i}$ such that

(i) $F_{i}\left(a_{1}, \ldots, a_{n+1}\right) \in \mathbf{p}_{i}$, 
(ii) $F_{i}\left(X_{1}, \ldots, X_{n+1}\right) \notin \mathbf{m} A\left[X_{1}, \ldots, X_{n+1}\right]$.

Setting $F\left(X_{1}, \ldots, X_{n+1}\right)=\prod_{i=1}^{k} F_{i}\left(X_{1}, \ldots, X_{n+1}\right)$, we have

(iii) $F\left(a_{1}, \ldots, a_{n+1}\right) \in \cap_{i=1}^{k} \mathbf{p}_{i}=(0)$,

(iv) $F\left(X_{1}, \ldots, X_{n+1}\right) \notin \mathbf{m} A\left[X_{1}, \ldots, X_{n+1}\right]$.

Thus no set of $(n+1)$-elements is $\mathbf{m}$-independent.

Thus it is established that gra $\leqslant$ sup a $\leqslant$ ht a. For prime ideals, using (1) of Proposition 5 and the fact that a system of parameters in a local ring is analytically independent, one knows that $\sup \mathbf{p}=\mathrm{ht} \mathbf{p}$, a fact which can be extended to radical ideals. It was first thought that perhaps sup always took one of the limiting values. The following examples show this to be false.

EXAMPLE. Let $A=k[X, Y, Z] /\left(X^{2}, X Y^{2}, X Y Z\right)=k[x, y, z], \mathbf{m}=(x, y, z)$, $\mathbf{a}=\mathbf{m}^{2}=\left(x y, x z, y^{2}, y z, z^{2}\right)$, and $\mathbf{b}=\left(x y, x z, y^{2}, y z\right)$. Then ht $\mathbf{a}=\mathrm{ht} \mathbf{m}$ $=2$ and $\operatorname{gr} \mathbf{a}=0$ since $0 \neq x y \in(0: \mathbf{a})$. Now $z^{2}$ is an a -independent element. For $c\left(z^{2}\right)^{n}=0$ implies that $c \in(x y) \subset \mathbf{a}$. So sup $\mathbf{a} \geqslant 1$. On the other hand, suppose $u, v \in \mathbf{a}$ are $\mathbf{a}$-independent. Since $x \mathbf{b}=0$, no elements of $\mathbf{b}$ are a-independent. Thus we can write $u=f(z) z^{p}+u^{\prime}, v=g(z) z^{q}+v^{\prime}$ where $u^{\prime}, v^{\prime} \in \mathbf{b}, p, q \geqslant 2$, and $f(0) \neq 0, g(0) \neq 0$. Assume with no loss of generality that $p \geqslant q$. Then $x g(z) T_{1}-x z^{p-q} f(z) T_{2}$ is a form which vanishes at $u, v$. However $x g(z) \equiv x g(0) \equiv x \not \equiv 0(\bmod a)$. Thus $u, v$ are not a-independent and $\sup \mathbf{a}=1$.

It is reasonable now to conjecture that sup can take any value between grade and height. The following easy result is useful in constructing sets of independent elements.

Proposition 7. Let $\mathbf{a}, \mathbf{b}$ be ideals of a ring $A, a_{1}, \ldots, a_{n} \in \mathbf{a}, \varphi: A \rightarrow A / \mathbf{b}$. If $\varphi\left(a_{1}\right), \ldots, \varphi\left(a_{n}\right)$ are $\varphi(\mathbf{a})$-independent, then $a_{1}, \ldots, a_{n}$ are $(\mathbf{a}+\mathbf{b})$-independent.

EXAMPLE. Let

$$
\begin{aligned}
A & =k\left[X_{0}, \ldots, X_{n}\right] /\left(X_{0}^{2}, X_{0} X_{1}^{2}, \ldots, X_{0} X_{1} \cdots X_{i-1} X_{i}^{2}, \ldots,\right. \\
& \left.X_{0} X_{1} \cdots X_{n-2} X_{n-1}^{2}, X_{0} X_{1} \cdots X_{n}\right) \\
& =k\left[x_{0}, \ldots, x_{n}\right],
\end{aligned}
$$

$\mathbf{m}=\left(x_{0}, \ldots, x_{n}\right)$. Clearly ht $\mathbf{m}=n$ and gr $\mathbf{m}=0$ since $0 \neq x_{0} x_{1} \cdots x_{n-1}$ $\in(0: \mathbf{m})$. Thus ht $\mathbf{m}^{k}=n, \mathrm{gr}^{k}=0$ for $k=1, \ldots, n+1$. The claim is that $\sup \mathbf{m}^{k} \geqslant n+1-k$ for $k=1, \ldots, n+1$ with equality holding when $k=1, n$, or $n+1$.

To verify the inequality, let $\mathbf{b}$ be the principal ideal generated by $x_{0} x_{1} \cdots x_{k-1}$ and

$\varphi: A \rightarrow A / \mathbf{b} \approx k\left[X_{0}, \ldots, X_{n}\right] /\left(X_{0}^{2}, X_{0} X_{1}^{2}, \ldots, X_{0} X_{1} \cdots X_{k-3} X_{k-2}^{2}\right.$,

$$
\left.X_{0} \cdots X_{k-1}\right) \text {. }
$$

Thus $\varphi\left(x_{k}^{k}\right), \ldots, \varphi\left(x_{n}^{k}\right)$ form an $(A / \mathbf{b})$-sequence and so are $\left(\varphi\left(x_{k}^{k}\right), \ldots, \varphi\left(x_{n}^{k}\right)\right)$ independent by Proposition 3. Hence $x_{k}^{k}, \ldots, x_{n}^{k}$ are $\left(x_{k}^{k}, \ldots, x_{n}^{k}\right.$, $\left.x_{0} x_{1} \cdots x_{k-1}\right)$-independent where $\left(x_{k}^{k}, \ldots, x_{n}^{k}, x_{0} x_{1} \cdots x_{k-1}\right) \subset \mathbf{m}^{k}$.

If $k=1$, sup $\mathbf{m}=\mathrm{ht} \mathbf{m}=n$. If $k=n+1$, then $x_{0} x_{1} \cdots x_{n-1} \in(0: \mathbf{m})$ $\subseteq\left(0: \mathbf{m}^{n+1}\right)$ but $x_{0} x_{1} \cdots x_{n-1} \notin \mathbf{m}^{n+1}$ so sup $\mathbf{m}^{n+1}=0$. If $k=n$, a modifi- 
cation of the previous example gives sup $\mathbf{m}^{n}=1$.

Whether the sequence of ideals in the above example can serve to confirm the conjecture on the intermediate values of sup is unclear. In general, the problem of imposing upper bounds on sup a (better then ht a) requires the construction of forms vanishing on arbitrary sets of elements of a. The usual constructions, for example, using determinants, tend to fail in this situation because the coefficients end up in a.

The fact that sup $\mathbf{p}=\mathrm{ht} \mathbf{p}$ for prime ideals $\mathbf{p}$ leads immediately to

Proposition 8. Let $A$ be a ring. The following conditions are equivalent:

(1) $\sup \mathbf{a}=\operatorname{gr}$ a for all ideals $\mathbf{a}$ of $A$;

(2) $\sup \mathbf{p}^{n}=\operatorname{gr} \mathbf{p}^{n}$ for all prime ideals $\mathbf{p}$ of $A$, all $n \geqslant 1$;

(3) $\sup \mathbf{p}=\operatorname{gr} \mathbf{p}$ for all prime ideals $\mathbf{p}$ of $A$;

(4) $A$ is Cohen-Macaulay.

If $\left(1^{\prime}\right)$ is the condition sup $\mathbf{a}=\mathrm{ht} \mathbf{a}$ for all ideals $\mathbf{a}$ of $A$ and $\left(2^{\prime}\right)$ is the condition sup $\mathbf{p}^{n}=h t \mathbf{p}^{n}$ for all prime ideals $\mathbf{p}$ and all $n \geqslant 1$, some natural next questions are to characterize those rings satisfying $\left(1^{\prime}\right)$ or $\left(2^{\prime}\right)$. It should be noted that $\left(2^{\prime}\right)$ implies sup $\mathbf{q}=\mathrm{ht} \mathbf{q}$ for all primary ideals. For the equality of (1) Proposition 5 is valid for p-primary ideals. Thus one can reduce to the case of a local ring and an m-primary ideal. In that case, $\mathbf{m}^{t} \subseteq \mathbf{q} \subseteq \mathbf{m}$ for some $t \geqslant 1$. Thus ht $\mathbf{q}=$ ht $\mathbf{m}^{t}=\sup \mathbf{m}^{t} \leqslant \sup \mathbf{q}$.

Definition 3. A ring $A$ is said to be $S_{n}(n=0,1,2, \ldots)$ if $\operatorname{depth} A_{\mathrm{p}}$ $\geqslant \min \left\{n, \operatorname{dim} A_{\mathbf{p}}\right\}$ for all $\mathbf{p} \in \operatorname{Spec} A$.

Here $\operatorname{depth} A_{\mathbf{p}}=\operatorname{gr} \mathbf{p} A_{\mathbf{p}}$. In other words, $A_{\mathbf{p}}$ is Cohen-Macaulay when ht $\mathbf{p} \leqslant n$ and gr $\mathbf{p} \geqslant n$ when ht $\mathbf{p}>n$. Clearly $A$ is $S_{1}$ if and only if every height 1 prime contains a regular element, or, if and only if the zero ideal of $A$ is unmixed.

Proposition 9. If $A$ is a ring in which sup $\mathbf{p}^{n}=\mathrm{ht} \mathbf{p}^{n}$ for all prime ideals $\mathbf{p}$ and all $n \geqslant 1$, then $A$ is $S_{1}$.

Proof. Suppose that $\mathbf{p}$ is an embedded prime of $(0)$. Then $\mathrm{ht} p \geqslant 1$ and $\mathbf{p}$ $=(0: x)$ for some $x$, where $x \notin \cap_{n} \geqslant 1 \mathbf{p}^{n}$. Choosing $n$ such that $x \in \mathbf{p}^{n-1}, x$ $\notin \mathbf{p}^{n}$, we get $x \in(0: \mathbf{p}) \subseteq\left(0: \mathbf{p}^{n}\right)$. Thus sup $\mathbf{p}^{n}=0$ whereas $h t \mathbf{p}^{n} \geqslant 1$, which is a contradiction.

\section{REFERENCES}

1. J. Barshay, Graded algebras of powers of ideals generated by A-sequences, J. Algebra 25 (1973), 90-99. MR 48 \#11074.

2. E. Boger, Minimalitatsbedingungen in der Theorie der Reduktionen von Idealen, Schr. Math. Inst. Univ. Münster No. 40 (1968), 57 pp. MR 39 \#200.

3. A. Micali, Sur les algèbres universelles, Ann. Inst. Fourier (Grenoble) 14 (1964), fasc. 2, 33-87. MR 31 \# 1275.

4. G. Valla, Elementi indipendenti rispetto ad un ideale, Rend. Semi. Mat. Univ. Padova 44 (1970), 339-354. MR 45 \#6802.

5. - Su certi isomorfismi di algebre e l'indipendenze rispetto ad un ideale, Matematiche (Catania) 26 (1971), 35-45 (1972). MR 47 \#4991.

6. O. Zariski and P. Samuel, Commutative algebra. Vol. 2, University Ser. in Higher Math., Van Nostrand, Princeton, N.J., 1960. MR 22 \#11006. 